\section{Cortical counting}

Neurons in the prefrontal cortex can sort visual stimuli into abstract categories. Following on from this, new research shows that prefrontal neurons can also sort visual images according to the number of stimuli they contain. Nieder et al. recorded from neurons in the monkey lateral prefrontal cortex while the animals carried out a task that involved judging relative numbers of dots, and found that many of the neurons were tuned for quantity, rather than for other visual features. this study were trained to perform a 'delayed match to quantity' task, in which they were shown two successive displays and had to respond if the two displays contained the same number of dots. The monkeys performed best when the numbers were small - as do humans, when they rapidly estimate quantities - but they could judge the relative numbers of items even if the sizes, shapes or arrangements of the dots differed.

More than a third of recorded neurons in the lateral prefrontal
The monkeys that were used in cortex were selective for stimulus number, regardless of the physical appearance of the stimuli. The neurons showed fairly broad, overlapping tuning curves, which could explain the improvement in performance that was seen when the displays were separated by a greater numerical distance.

Evidence that the activity of these neurons is important for behaviour comes from error trials. When the monkeys responded inaccurately, the neural activity for the preferred quantity was significantly less than on successful trials. Although it is easy to imagine ways in which numerical judgements would be useful in the wild - for example, when deciding whether to fight a smaller group of foes or to flee from a larger group it remains to be seen what this kind of test situation can tell us about more natural behaviours.

Rachel Jones

(2) References and links ORIGINAL RESEARCH PAPER Nieder, A. et al. Representation of the quantity of visual items in the Representation of the quantity of visual i. 1708-1711 (2002)

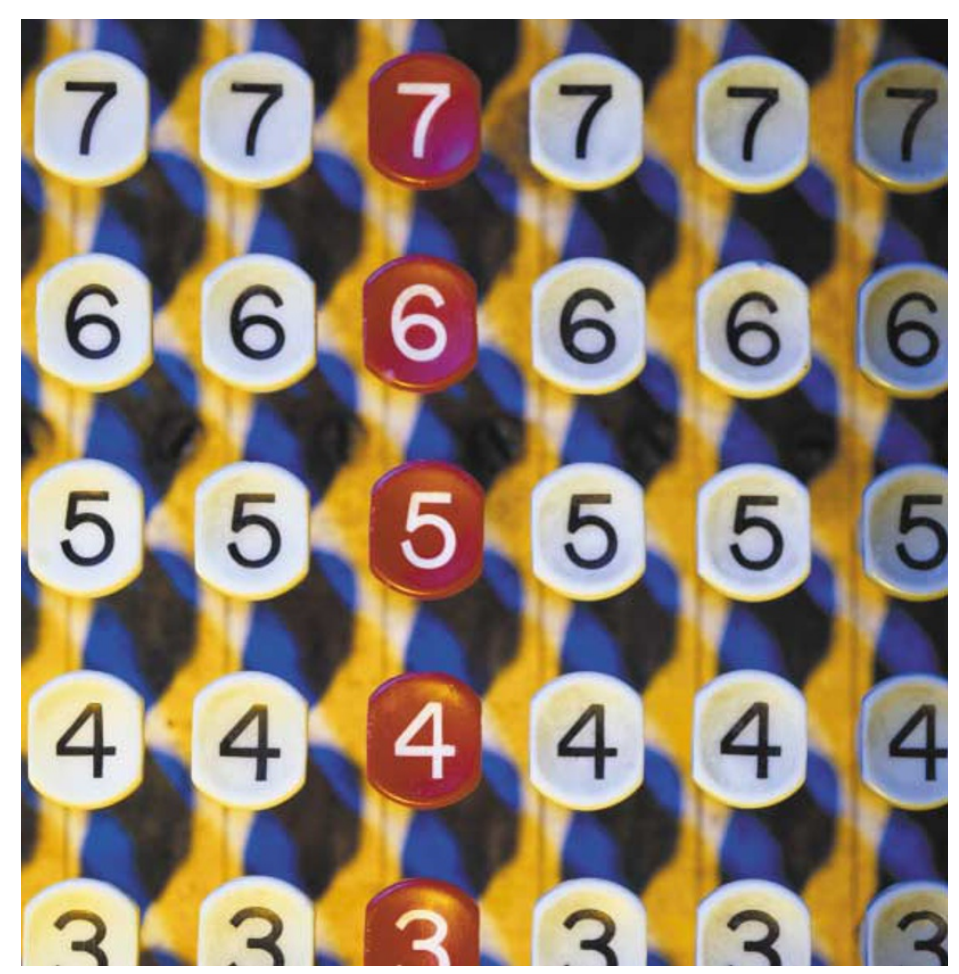

Regeneration of hippocampal pyramidal neurons after ischemic brain injury by recruitment of endogenous neural progenitors.

Nakatomi, H. et al. Cell 110, 429-441 (2002)

The use of neural stem cells to repair the brain after injury is a tantalizing possibility, and this paper provides good evidence that such cells can indeed fulfil their potential. After an ischaemic lesion, periventricular and parenchymal hippocampal progenitors can give rise to neurons in the adult rat brain. The infusion of fibroblast and epidermal growth factors can potentiate this regenerative capacity, and the new neurons can form functional circuits and promote behavioural recovery.

Directed differentiation of embryonic stem cells into motor neurons.

Wichterle, H. et al. Cell 110, 385-397 (2002)

We know many factors that participate in the generation of spinal motor neurons during ontogeny, and the authors set out to recapitulate this developmental programme by directing embryonic stem cells to become motor neurons. Motor neurons obtained in this way integrated into the embryonic spinal cord and proceeded to innervate muscle. So, our understanding of the mechanisms that govern neural development can be used to direct stem cells into specific neuronal populations, opening a series of possibilities for their therapeutic use.

LeX/ssea-1 is expressed by adult mouse CNS stem cells, identifying them as nonependymal.

Capela, A. \& Temple, S. Neuron 35, 865-875 (2002)

Identifying specific markers of adult stem cells would aid in their characterization and enrichment. Capela and Temple report that the subventricular stem cells of the adult mouse express the Lewis X (LeX) carbohydrate, and show that purified LeX-positive cells are indeed stem cells, indicating that this carbohydrate might be a useful marker of this cell type. Moreover, the authors solve a controversy in the field by showing that LeX-positive stem cells are not ependymal cells.

Cell-intrinsic differences between stem cells from different regions of the peripheral nervous system regulate the generation of neural diversity.

Bixby, S. et al. Neuron 35, 643-656 (2002)

Although environmental cues are crucial for determining the ultimate fate of neural stem cells, the role of cell-intrinsic differences has been largely neglected. Stem cells of the neural crest normally give rise to neurons in the gut and to glia in nerves, and Bixby et al. exploited this difference to test whether cell fate was determined by the initial nature of the stem cell. Depending on their origin, stem cells did show intrinsic differences in their responsiveness to gliogenic and neurogenic factors, and transplantation of gut- and nerve-derived stem cells into nerves gave rise to neurons and glia, respectively. These observations highlight the importance of intrinsic factors in determining the fate of a stem cell. 